\title{
Using Coulombian Approach for Modeling Scalar Potential and Magnetic Field of a Permanent Magnet With Radial Polarization
}

\author{
H. L. Rakotoarison, J.-P. Yonnet, and B. Delinchant \\ Laboratoire d'Electrotechnique de Grenoble, Saint Martin d'Hères 38402, France
}

\begin{abstract}
New compact semianalytical expressions of the scalar potential and magnetic fields produced by a radially polarized permanent magnet are described in this paper by using Coulombian approach. It uses fictitious magnetic charge to model the magnetic field intensity. With this fast model, we can compute the demagnetization field in each point inside the permanent magnet and the magnetic fields outside it.
\end{abstract}

Index Terms-Analytical model, magnetic field, radial polarized magnet.

\section{INTRODUCTION}

$\mathbf{R}$ ADIAL polarized sector permanent magnets are often used in electromagnetic device. Most applications of this type of magnet were found in electrical motor and no-contact magnetic torque transmitter [1]. Recently, new application of this type of magnet is encountered in diamagnetic levitation device, used for microparticle sorting [2], 3-D force sensor [3], and accelerometer; those applications lead us to realize this investigation.

For cuboïdal magnets with a uniform magnetization, analytical expression can be used to calculate the magnetic field and even the forces created between two magnets [4]. However, for arc-shaped magnets with radial magnetization, the magnetic field calculation is more difficult.

Furlani [5] had proposed the first 3-D semianalytical model of flux density, using amperian approach, but the expressions of $B_{r}$ and $B_{\theta}$ need the sum of two integrals computation, which can be time consuming if the model is used for dynamic simulation or optimization process. This paper proposes a compact magnetic field modeling with single numerical integration.

The final result is helpful if a continuous model of the magnetic field is required and demagnetization study is needed.

We suppose that the magnet's polarization is rigid and uniform. The magnetic field is only generated by the arc-shaped magnet. If the real device is made with more than one sector, the field can be obtained by addition of the different contributions.

\section{Electrostatic and Magnetostatic Analogy}

\section{A. Basic Equations}

Let us start from the four Maxwell's equations in electrostatic [(1) and (2)] and magnetostatic [(3) and (4)]

$$
\begin{aligned}
\vec{\nabla} \times \vec{E} & =\overrightarrow{0} \\
\vec{\nabla} \cdot \vec{D} & =\rho
\end{aligned}
$$

$$
\begin{aligned}
\vec{\nabla} \times \vec{H} & =\vec{j} \\
\vec{\nabla} \cdot \vec{B} & =0
\end{aligned}
$$

where $E, D, H, B, \rho$, and $j$ are electric field, electric flux density, magnetic field intensity, magnetic flux density, electrical charge, electric current density, respectively.

The magnetic field is only produced by the magnet, so (3) becomes

$$
\vec{\nabla} \times \vec{H}=\overrightarrow{0}
$$

Taking the material properties into account

$$
\begin{aligned}
& \vec{D}=\varepsilon_{0} \vec{E} \\
& \vec{B}=\mu_{0} \vec{H}+\vec{J}
\end{aligned}
$$

$\varepsilon_{0}$ and $\mu_{0}$ are, respectively, the permittivity and the permeability of the vacuum, and $J$ is the polarization of the magnet.

By using (6) and (7), expression (2) and (4) become, respectively, (8) and (9)

$$
\begin{aligned}
& \vec{\nabla} \cdot \vec{E}=\frac{\rho}{\varepsilon_{0}} \\
& \vec{\nabla} \cdot \vec{H}=-\frac{\vec{\nabla} \cdot \vec{J}}{\mu_{0}} .
\end{aligned}
$$

Equations (1) and (8) show the duality between electrical field and electrical charge density $\rho$ in electrostatic problem.

If we introduce the fictitious magnetic charge density $\sigma$ and by applying the same duality as in (1) and (8) between magnetic field and fictitious magnetic charge in magnetostatic equations (5) and (9), we can write

$$
\vec{\nabla} \cdot \vec{H}=\frac{\sigma}{\mu_{0}} .
$$

By identification with (9), the fictitious charge density is

$$
\sigma=-\vec{\nabla} \cdot \vec{J}
$$

This fictitious magnetic charge density $\sigma$ is composed by a volume charge density $\sigma_{v}$ and surface charge density $\sigma_{s}$ (Fig. 1). 


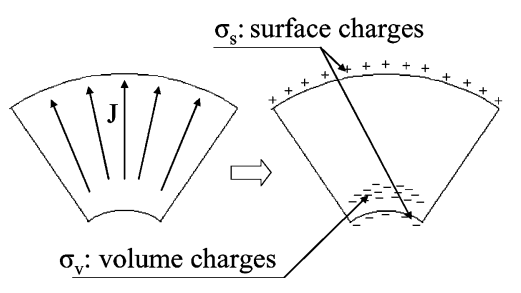

Fig. 1. Upper view of the magnet, showing its model with fictitious surface and volume charges.

If the magnetic polarization $J$ is uniform and radial, the expression of $\sigma_{v}$ in cylindrical coordinate system $(r, \theta, z)$ is

$$
\sigma_{v}=-\vec{\nabla} \cdot \vec{J}=-\frac{J}{r}
$$

Finding the surface charge density $\sigma_{s}$ is straightforward; it can be obtained by referencing (4), which means that, considering the entire volume of the magnet, there was no monopole magnetic charge, so

$$
\iint_{s} \sigma_{s} d s+\iiint_{v} \sigma_{v} d v=0
$$

$\vec{n}$ defines the unit vector normal to surface; by using (12) and applying Green's theorems, after identification, we obtain

$$
\sigma_{s}=\vec{J} \cdot \vec{n} .
$$

\section{B. Electrostatic Equivalent Model}

Finally, the electrostatic equivalent model of the permanent magnet with radial polarization can be illustrated as in Fig. 1. By projecting the polarization into the six surfaces of a magnet, the surface charge densities exist only in the inner and outer surface of the magnet.

The volume charge decreases according to a $1 / r$ function from the inner to the outer surface. From (5), we can proof the existence of the magnetic scalar potential $\varphi$ with

$$
\vec{H}=-\vec{\nabla} \cdot \varphi .
$$

Basic expression for scalar potential and field calculation produced by the permanent magnet are obtained in (16) and (17), by using the electrostatic approach

$$
\varphi_{P}=\frac{1}{4 \pi \mu_{0}}\left(\iint_{s} \cdot \frac{\sigma_{s}}{\left|\vec{r}-\vec{r}^{\prime}\right|} d s+\iiint_{v} \frac{\sigma_{v}}{\left|\vec{r}-\vec{r}^{\prime}\right|} d v\right) .
$$

By applying (15), the magnetic field intensity is

$$
\begin{aligned}
\vec{H}_{P}=\frac{1}{4 \pi \mu_{0}} & \left(\iint_{s} \cdot \frac{\sigma_{s}}{\left|\vec{r}-\vec{r}^{\prime}\right|^{3}}\left(\vec{r}-\vec{r}^{\prime}\right) d s\right. \\
& \left.+\iiint_{v} \frac{\sigma_{v}}{\left|\vec{r}-\vec{r}^{\prime}\right|^{3}}\left(\vec{r}-\vec{r}^{\prime}\right) d v\right) .
\end{aligned}
$$

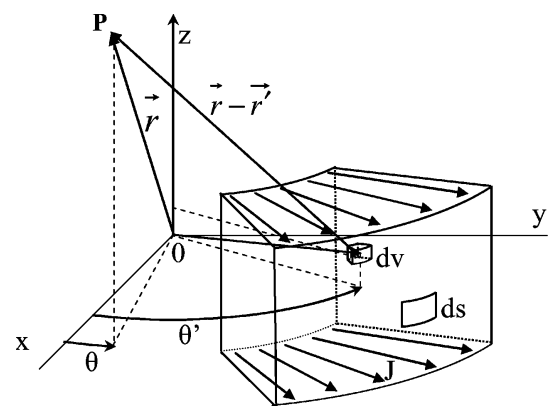

Fig. 2. Computing magnetic field produced by surface and volume charge density on a point $P$ in cylindrical coordinate.

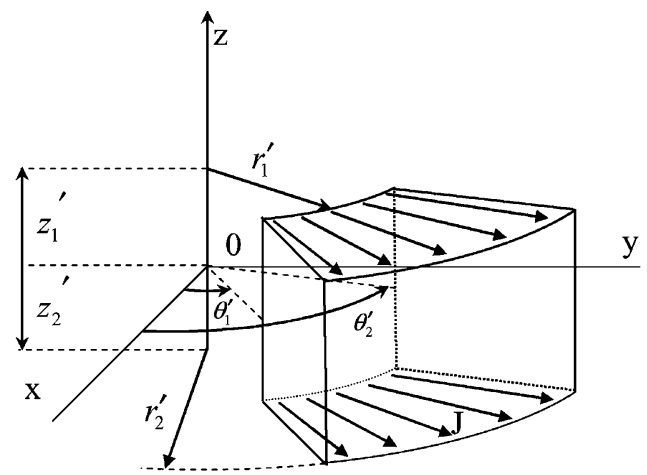

Fig. 3. Parameterization of the magnet.

The radii $\vec{r}$ and $\vec{r}^{\prime}$ define the observation point $P$ position and its distance to the elementary field source enclosed by $d v$ or $d s$ (Fig. 2).

\section{SCALAR POTENTIAL}

After integrating (16) analytically, we obtain a single numerical integration with the parameterization shown in Fig. 3

$\varphi(r, \theta, z)=\frac{J}{4 \pi \mu_{0}} \cdot \int_{\theta_{1}^{\prime}}^{\theta_{2}^{\prime}} \sum_{i=1}^{2} \sum_{j=1}^{2}(-1)^{i+j} d \varphi\left(r, \theta, z, r_{i}^{\prime}, \theta^{\prime}, z_{j}^{\prime}\right) d \theta^{\prime}$.

By using $\alpha=\theta^{\prime}-\theta$ with $\theta_{1}^{\prime} \leqslant \theta^{\prime} \leqslant \theta_{2}^{\prime}$ and

$$
\begin{aligned}
& \left.G(\alpha)=\sqrt{(} r-r_{i}^{\prime} \cos (\alpha)\right)^{2}+\left(r_{i}^{\prime} \sin (\alpha)\right)^{2}+\left(z-z_{j}^{\prime}\right)^{2} \\
& \begin{aligned}
d \varphi\left(r, \theta, z, r_{i}^{\prime}, \theta^{\prime}, z_{j}^{\prime}\right) \\
\quad=\left(z-z_{j}^{\prime}\right)\left(1-\ln \left(r_{i}^{\prime}\right.\right. \\
\quad-r \cos (\alpha)+G(\alpha)))-\left(r_{i}^{\prime}-r \cos (\alpha)\right) \ln \left(\left(z-z_{j}^{\prime}\right)\right. \\
\quad+G(\alpha))+r_{i}^{\prime} \ln \left(\left(z_{j}^{\prime}-z\right)+G(\alpha)\right) \\
\quad+r \sin (\alpha)\left[\arctan \left(\frac{\left(z_{-} z_{j}^{\prime}\right)\left(r_{i}^{\prime}-r \cos (\alpha)\right)}{r \sin (\alpha) G(\alpha)}\right)\right. \\
\left.-\arctan \left(\frac{\left(z-z_{j}^{\prime}\right)}{r \sin (\alpha)}\right)\right] .
\end{aligned}
\end{aligned}
$$

\section{MAGNETIC FiELd EXPRESSION}

After defining the magnetic scalar potential of the magnet, we can express the three components of the field by using (15). 
TABLE I

GeOMETRICAL SPECIFICATIONS OF THE PERMANENT MAGnet

\begin{tabular}{lll}
\hline \hline \multicolumn{1}{c}{ Symbols } & \multicolumn{1}{c}{ Parameters } & Values \\
\hline r'1 & Inner radius & $1 \mathrm{~mm}$ \\
r'2 & Outer radius & $4 \mathrm{~mm}$ \\
$\theta^{\prime} 1$ & Start angle & $-\pi / 8$ radian \\
$\theta^{\prime} 2$ & End angle & $\pi / 8$ radian \\
z'1, z'2 & Half height & $0.5 \mathrm{~mm}$ \\
\hline \hline
\end{tabular}

\section{A. Radial Component of the Field}

Formula of the radial field component is

$H_{r}(r, \theta, z)=\frac{J}{4 \pi \mu_{0}} \cdot \int_{\theta_{1}^{\prime}}^{\theta_{2}^{\prime}} \sum_{i=1}^{2} \sum_{j=1}^{2}(-1)^{i+j} d H_{r}\left(r, \theta, z, r_{i}^{\prime}, \theta^{\prime}, z_{j}^{\prime}\right) d \theta^{\prime}$.

$d H_{r}$ can be written as follows:

$$
\begin{aligned}
& d H_{r}\left(r, \theta, z, r_{i}^{\prime}, \theta^{\prime}, z_{j}^{\prime}\right) \\
& =\frac{-r_{i}^{\prime}\left(z-z_{j}^{\prime}\right)\left(r-r_{i}^{\prime} \cos (\alpha)\right)}{\left[\left(r-r_{i}^{\prime} \cos (\alpha)\right)^{2}+\left(r_{i}^{\prime} \sin (\alpha)\right)^{2}\right] \cdot G(\alpha)} \\
& \quad+\sin (\alpha) \arctan \left[\frac{\left(z-z_{j}^{\prime}\right)\left(r_{i}^{\prime}-r \cos (\alpha)\right)}{(r \sin (\alpha)) \cdot G(\alpha)}\right] \\
& \quad-\cos (\alpha) \ln \left[G(\alpha)-\left(z-z_{j}^{\prime}\right)\right] .
\end{aligned}
$$

\section{B. Azimuthal Component}

$H_{\theta}(r, \theta, z)=\frac{J}{4 \pi \mu_{0}} \cdot \int_{\theta_{1}^{\prime}}^{\theta_{2}^{\prime}} \sum_{i=1}^{2} \sum_{j=1}^{2}(-1)^{i+j} d H_{\theta}\left(r, \theta, z, r_{i}^{\prime}, \theta^{\prime}, z_{j}^{\prime}\right) d \theta^{\prime}$

with

$$
\begin{aligned}
& d H_{\theta}\left(r, \theta, z, r_{i}^{\prime}, \theta^{\prime}, z_{j}^{\prime}\right) \\
& =\frac{-r_{i}^{\prime}\left(z-z_{i}^{\prime}\right)\left(r_{i}^{\prime} \sin (\alpha)\right)}{\left[\left(r-r_{i}^{\prime} \cos (\alpha)\right)^{2}+\left(r_{i}^{\prime} \sin (\alpha)\right)^{2}\right] \cdot G(\alpha)} \\
& \quad-\cos (\alpha) \arctan \left[\frac{\left(z-z_{i}^{\prime}\right)\left(r_{i}^{\prime}-r \cos (\alpha)\right)}{(r \sin (\alpha)) \cdot G(\alpha)}\right] \\
& \quad-\sin (\alpha) \ln \left[G(\alpha)-\left(z-z_{i}^{\prime}\right)\right] .
\end{aligned}
$$

\section{Axial Component}

$H_{z}(r, \theta, z)=\frac{J}{4 \pi \mu_{0}} \cdot \int_{\theta_{1}^{\prime}}^{\theta_{2}^{\prime}} \sum_{i=1}^{2} \sum_{j=1}^{2}(-1)^{i+j} d H_{z}\left(r, \theta, z, r_{i}^{\prime}, \theta^{\prime}, z_{j}^{\prime}\right) d \theta^{\prime}$

with

$$
\begin{aligned}
d H_{z}\left(r, \theta, z, r_{i}^{\prime}, \theta^{\prime}, z_{j}^{\prime}\right)= & \frac{r_{i}^{\prime}}{G(\alpha)}-\ln \left[\left(r_{i}^{\prime}-r \cos (\alpha)\right)+G(\alpha)\right] \\
& +\frac{1}{2} \ln \left[(r \sin (\alpha))^{2}+\left(z-z_{i}^{\prime}\right)^{2}\right] .
\end{aligned}
$$

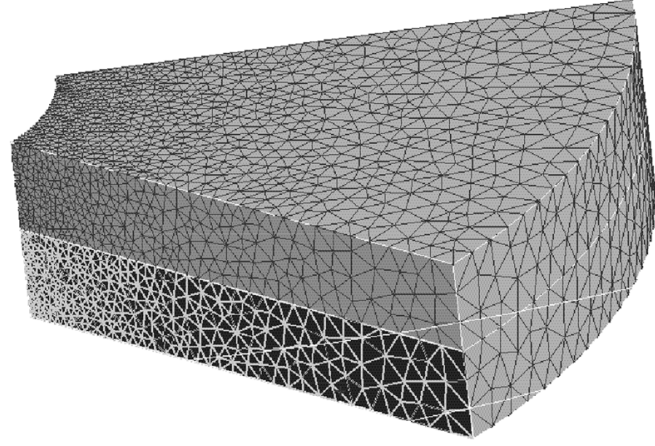

$L^{z} \times$

Fig. 4. Mesh used for the finite element analysis.

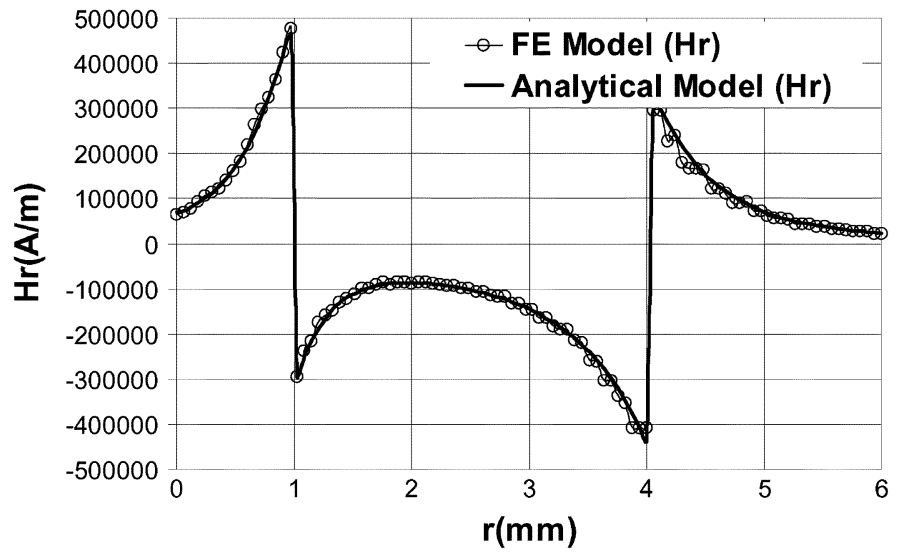

Fig. 5. Radial component of the filed from $r=0 \mathrm{~mm}$ to $r=6 \mathrm{~mm}$, with $z=$ $0 \mathrm{~mm}$ and $\theta=0^{\circ}$

\section{Results AND Finite ElEMENT Modeling}

As validation of the model, a finite element analysis was done by using FLUX3D software [6].

We have chosen the dimensions given in Table I, and we have taken $J=1 T$.

For obtaining a precise result in finite element analysis, the mesh close to the inner radius of the sector must be very fine (Fig. 4).

\section{A. Discontinuity of the Normal Component of the Magnetic Field Intensity}

Fig. 5 shows the discontinuity of radial field (the tangential component) inside and outside the magnet ( $r$ varying from 0 to $6 \mathrm{~mm}$ ); it illustrates as well that the demagnetization effect is less important near the inner radius $(1 \mathrm{~mm})$ than close to the outer radius $(4 \mathrm{~mm})$. This feature can contribute to the design parameters during sizing process.

\section{B. Comparing the Azimuthal Component}

This investigation allows us to check the continuity of the normal component (Fig. 6) of the magnetic field intensity. 


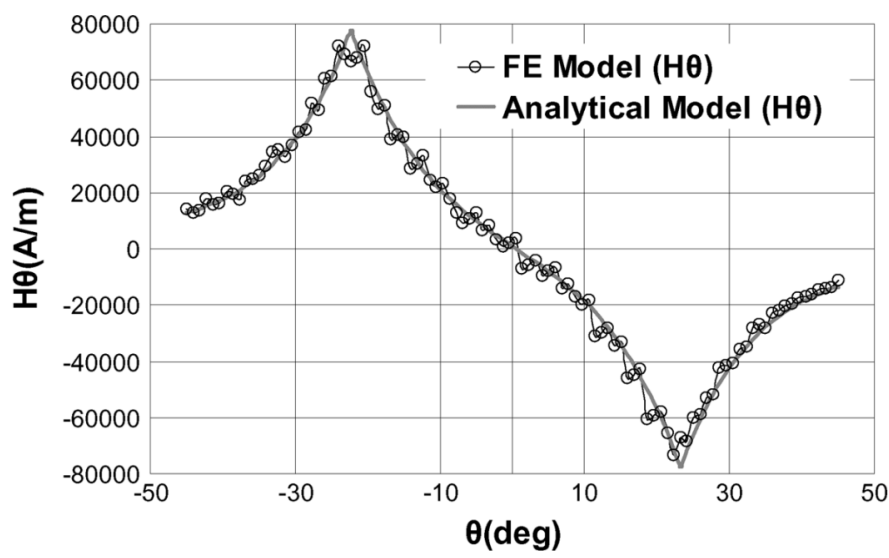

Fig. 6. Azimuthal component of the field from $\theta=-45^{\circ}$ to $\theta=45^{\circ}$, with $r=2.5 \mathrm{~mm}$ and $z=0 \mathrm{~mm}$.

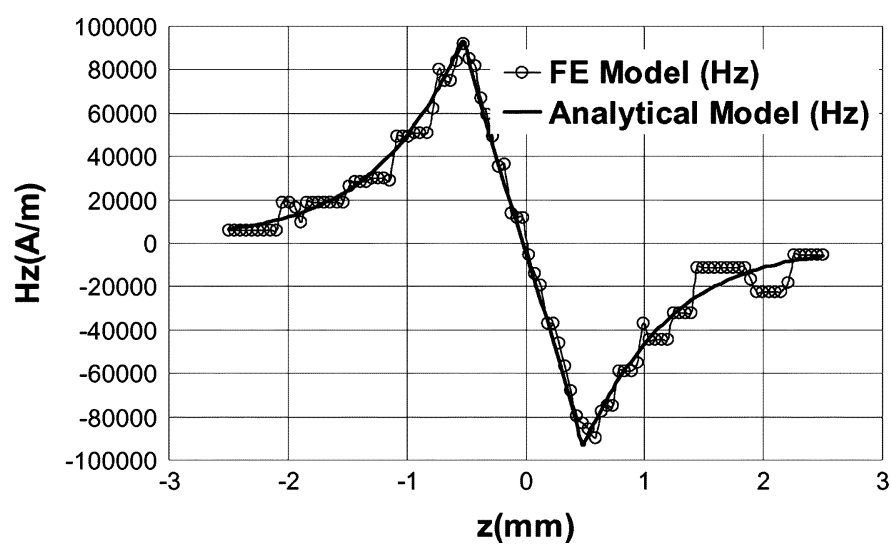

Fig. 7. Axial component of the field from $z=-2.5 \mathrm{~mm}$ to $z=2.5 \mathrm{~mm}$, with $r=2.5 \mathrm{~mm}$ and $\theta=0^{\circ}$

\section{Comparing the Axial Component}

As the azimuthal component, the behavior of the axial component (Fig. 7) is continued, because it is the normal component of the magnetic field intensity.

\section{Magnetic Field Radiated by a Cylinder Magnet}

The distribution of the modulus of the magnetic field radiated by a ring-shaped magnet is depicted in Fig. 8. The polarization remains radial. Let us remark the presence of a hole of field in

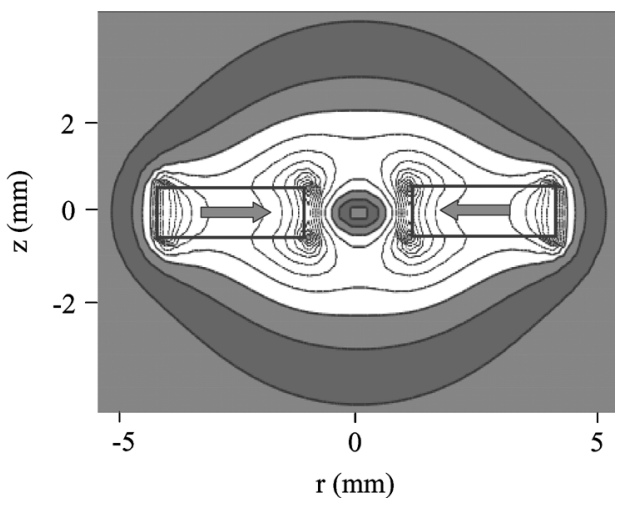

Fig. 8. Cross-section view of the field radiated by a right-shaped magnet with a radial polarization.

the center of the magnet; this feature is significant if diamagnetic levitation is required [3].

\section{CONCLUSION}

This article proposes a fast 3-D semianalytical expression of the scalar potential and the magnetic field produced by an arcshaped permanent magnet with radial polarization. The result can also be used for ring-shaped magnet. Expressions of the scalar potential and the magnetic field intensity is detailed and compared with a finite element method (FEM) simulation.

\section{REFERENCES}

[1] P. Elies and G. Lemarquand, "Analytical optimization of the torque of a permanent-magnet coaxial synchronous coupling," IEEE Trans. Magn., vol. 34, no. 4, pp. 2267-22731, Jul. 1998.

[2] H. Chetouani, C. Jeandey, V. Haguet, H. Rostaing, C. Dieppedale, and G. Reyne, "Diamagnetic levitation with permanent magnets for contactless guiding and trapping of microdroplets and particles in air and liquids," IEEE Trans Magn., vol. 42, no. 10, pp. 3557-3559, Oct. 2006.

[3] M. Boukallel, E. Piat, and J. Abadie, "Passive diamagnetic levitation: Theoretical foundations and application to the design of a micro-nano force sensor," in Proc. IEEE/RSJ Int. Conf. Intell. Robot. Syst., Oct. 2003, vol. 1, pp. 529-534.

[4] G. Akoun and J. P. Yonnet, "3D analytical calculation of the forces exerted between two cuboidal magnets," IEEE Trans. Magn., vol. MAG-20, no. 5, pp. 1962-1964, Sep. 1984.

[5] E. P. Furlani, S. Reznik, and A. Kroll, "A three-dimensional field solution for radially polarized cylinders," IEEE Trans. Magn., vol. 31, no. 1, pp. 844-851, Jan. 1995.

[6] Copyright Cedrat Research, "FLUX3D," Meylan Cedex, France.

Manuscript received January 30, 2006 (e-mail: lalao.rakotoarison-harijaona@leg.ensieg.inpg.fr). 